\title{
Note on a Parametric Relation for Separating Flow over a Rough Hill
}

\author{
J. B. R. Loureiro • A. P. Silva Freire
}

Received: 13 February 2008 / Accepted: 9 February 2009 / Published online: 5 March 2009

(C) Springer Science+Business Media B.V. 2009

\begin{abstract}
A parametrization method used to account for the effects of flow separation and wall roughness on the lower boundary condition for turbulent boundary layers is investigated against direct numerical simulation and laser Doppler anemometry data. The numerical simulation represents flow over a smooth, flat surface with a prescribed external adverse pressure gradient. The water-channel experiments cover flow over smooth and rough hills for two specified Reynolds numbers. Global optimization algorithms based on four different direct search methods are used to assess the parametrization function, $C$, in terms of local mean velocity profiles and the parametrization parameters $u_{*}$ (friction velocity), $\partial_{x} p$ (local pressure gradient), $z_{0}$ (effective roughness) and $d$ (zero-plane displacement). The study investigates regions of attached and reversed flows, and forty-two velocity profiles are compared with the proposed expression for the function $C$, including two profiles that satisfy the solution of Stratford.
\end{abstract}

Keywords Hill flow $\cdot$ Lower boundary condition $\cdot$ Roughness $\cdot$ Separation

\section{Introduction}

Any description of turbulent flow over natural land should ideally contemplate two very difficult modelling issues: wall roughness and flow separation. In particular, since these two characteristics often occur simultaneously, their effects should be considered together and not separately.

J. B. R. Loureiro $(\bowtie) \cdot$ A. P. Silva Freire

Programa de Engenharia Mecânica (COPPE/UFRJ), Universidade Federal do Rio de Janeiro, C.P. $68503,21945-970$ Rio de Janeiro, Brazil

e-mail: jbrloureiro@gmail.com

J. B. R. Loureiro

Divisão de Metrologia Científica, Instituto Nacional de Metrologia (INMETRO),

22.050-050 Rio de Janeiro, Brazil 
Unfortunately, this has not been the practice to date. The excessive level of complexity involved in the description of both subjects has usually forced workers to approach the problem individually. Recently, Loureiro et al. (2008) proposed a new treatment for the lower boundary condition that, in principle, can be used in regions of attached and separated flows over rough surfaces. The specification of wall functions to represent near-wall turbulence is particularly helpful for flows over rough surfaces, since the fine-scale complexities generated by the flow around roughness elements cannot be resolved even in the present day environment of optimized algorithms and high computing power.

The treatment of Loureiro et al. (2008) is not susceptible to the deficiencies encountered by the classical logarithmic law of the wall. Close to a separation point, the proposed solution reduces to the square-root law of Stratford (1959). In particular, the relevant scales of the flow are arranged so that far away from a separation point the skin friction velocity $\left(u_{*}=\sqrt{\tau_{w} / \rho}\right.$, $\tau_{w}=$ local wall shear stress) is the prescribed scale of the flow, whereas near to a separation point a new scale based on the local pressure gradient is introduced $\left(u_{p}=\left(\left(z_{0} / \rho\right) \partial_{x} p\right)^{1 / 2}\right.$, where $z_{0}=$ effective roughness, $\partial_{x} p=$ local pressure gradient).

One major difficulty with all derivations of the law of the wall is the appearance of an additive parameter that must be determined experimentally. The physical modelling of this parameter is necessarily very complex for it should incorporate, for example, the diversity of details that define a rough wall. Undaunted by this prospect, researchers have striven to develop simplified theories that can be applied to particular conditions. The natural consequence is that a large body of empirical data can be found in the literature that characterizes the surface parameters. Many specific texts on flow over roughness could be cited here; instead, we refer the reader to Oke (1987), Garratt (1992) and Kaimal and Finnigan (1994).

In Loureiro et al. (2008), the additive parameter of the law of the wall formulation, $C$, is parametrized by considering the limiting form of the proposed solution as $\tau_{w} \gg(1 / \rho) \partial_{x} p$. The implication is that $C$ is written in terms of $u_{*},(1 / \rho) \partial_{x} p, z_{0}$ and the von Kármán constant, $\varkappa(=0.4)$. The clear attractiveness of this approach is the possibility of defining $C$ from, as mentioned before, the vast collection of $z_{0}$ values that appears in literature. The apparent weakness, on the other hand, is the lack of support of the theory in regions where the above inequality is not satisfied, that is, close to a separation point and in regions of reverse flow.

The purpose of the present work is to investigate in detail the validity of the new treatment of Loureiro et al. (2008) for the lower boundary condition through direct numerical simulation (DNS) and experimental data. In particular, the behaviour of the additive parameter $C$ in regions of attached as well as separated flow is investigated with the help of global optimization algorithms based on direct search methods. The parametrization of $C$ proposed by Loureiro et al. (2008) is also scrutinized in greater detail in regions close to a separation point and in regions of reverse flow. Four sets of data are considered here: the smooth wall DNS dataset of $\mathrm{Na}$ and Moin (1998), the smooth wall laser Doppler anemometry (LDA) dataset of Loureiro et al. (2007a, b) and the two rough wall LDA datasets of Loureiro et al. (2008). In particular, one of the datasets of Loureiro et al. (2008) permits $C$ to be evaluated at a point of separation over a rough wall.

\section{Theory}

In Loureiro et al. (2008) the lower boundary condition is specified through

$$
u=2 \varkappa^{-1} \sqrt{\Delta_{w}}+\varkappa^{-1} u_{*} \ln \left(\left(\sqrt{\Delta_{w}}-u_{*}\right) /\left(\sqrt{\Delta_{w}}+u_{*}\right)\right)+C,
$$


where $u$ is the longitudinal velocity component, $\Delta_{w}=\rho^{-1} \tau_{w}+\left(\left(z_{0} / \rho\right) \partial_{x} p\right) z^{+}, z^{+}=$ $(z-d) / z_{0}, z$ is the vertical distance from the bottom of the roughness elements, $d=$ zeroplane displacement, $z_{0}=$ effective roughness, $\tau_{w}=$ local wall shear stress, $u_{*}=\sqrt{\tau_{w} / \rho}, \partial_{x}$ $p=$ local pressure gradient at the wall and $C$ is a parameter that depends on the flow and wall conditions.

For a detailed description of the conditions that lead to Eq. 1, including its context within the asymptotic theory of turbulent boundary layers, see Cruz and Silva Freire (1998, 2002) and Loureiro et al. (2008). Equation 1 is obtained from first principles through a double integration of a local motion equation together with the mixing length hypothesis, and is not obtained through an arbitrary interpolation procedure based on empirical arguments. It is an exact asymptotic result.

Specifically, Eq. 1 is valid in the inertial sublayer (IS), a region (Cheng and Castro 2002) "within which the Monin-Obukhov similarity theory is applicable, the flow is horizontally homogeneous (at least on the scale of the roughness element spacing), the turbulent statistics are roughly constant with height, and the mean wind profile is logarithmic". In fact, Loureiro et al. (2008) have shown that Eq. 1 is not only valid in regions of attached flow, where the velocity profile is logarithmic, but also in regions of separated flow where a Stratford velocity profile must hold. In the relevant limiting cases $\left(\partial_{x} p\right) z \ll \tau_{w}$ and $\left(\partial_{x} p\right) z \gg \tau_{w}$, Eq. 1 can be easily verified to reduce to the classical law of the wall and to the solution of Stratford (1959) respectively (see analysis below).

Most surfaces need, for a complete characterization of their roughnesses, the specification of two parameters: the zero-plane displacement, $d$, and the effective roughness, $z_{0}$. Provided these two quantities are known, parameters $u_{*}, \partial_{x} p$ and $C$ can be estimated through Eq. 1 from given profiles of $u$ versus $z$. However, if Eq. 1 is to be intended as a lower boundary condition, $C$ needs to be modelled in terms of the other flow parameters.

To model $C$, diverse arguments could be summoned. Frequently, scale analysis together with empirical data are used to define the functional behaviour of unknown parameters. In the present case, mathematical consistency between Eq. 1 and the classical law of the wall, $u=\left(u_{*} / \varkappa\right) \ln z^{+}$, is required. Indeed, in the limiting case $\left(\partial_{x} p\right) z \ll \tau_{w}$, Eq. 1 reduces to the logarithmic expression

$$
u=\left(u_{*} / \varkappa\right) \ln \left[\left(\left(z_{0} / \rho\right)\left(\partial_{x} p\right)\right)\left(4 u_{*}^{2}\right)^{-1} z^{+}\right]+(2 / \varkappa) u_{*}+C .
$$

Near a point of separation where, on the other hand, $\left(\partial_{x} p\right) z \gg \tau_{w}$, Eq. 1 reduces to

$$
u=(2 / \varkappa)\left[\left(z_{0} / \rho\right)\left(\partial_{x} p\right) z^{+}\right]^{1 / 2}+C,
$$

viz. the solution of Stratford (1959).

To define $C$, Loureiro et al. (2008) require that Eq. 2 be consistent with the classical law of the wall, that is,

$$
C=\varkappa^{-1} u_{*}\left[\ln \left(4 u_{*}^{2} /\left(\left(z_{0} / \rho\right) \partial_{x} p\right)\right)-2\right],
$$

where Eq. 4 is strictly valid for attached flows in neutral conditions. At a separation point, Eq. 4 furnishes $C=0$. This result is, as we shall see, inconsistent with Loureiro et al. (2008) for flow over a rough wall.

The obvious implication is that Eq. 4 needs to be specialized in regions where $\tau_{w} \approx 0$, which is by no means a simple task. While many studies can be found in the literature on separated flows (see, e.g., the review paper of Simpson (1996) for a large collection of references), very few deal with flow over rough surfaces and pay attention to the flow features at or near a 
separation point. The work of Simpson (1996) is a typical example: no mention of rough surfaces or of the solution of Stratford is made. Of course, roughness and separation effects are particularly relevant in micrometeorological applications. Most contributions to this subject, however, have focused on other issues including velocity speed-up and scalar dispersion; see, e.g., Britter et al. (1981), Snyder and Britter (1987), Aya et al. (1987), Athanassiadou and Castro (2001) and Ohba et al. (2002).

In a different guise, the onset of separation in turbulent, neutral flow over hills was studied in Wood (1995). By extrapolating two-dimensional linear results and considering a logarithmic undisturbed velocity profile, a simple algebraic equation was developed to estimate the approximate critical slope of a hill $\left(\theta_{\text {crit }}\right)$ for which the flow first separates. The resulting expression for $\theta_{\text {crit }}$ conceives a dependency of the critical slope on three characteristic lengths - the height scales of the inner $(l)$ and shear dominated middle $\left(h_{m}\right)$ layers and the effective roughness $\left(z_{0}\right)$ - but not on the exact shape of the hill. Despite this difficulty, comparisons of $\theta_{\text {crit }}$ with numerical predictions and observations for two-dimensional flows show good qualitative agreement. Predictions become especially inaccurate when hills present a strong streamwise asymmetry or three-dimensional effects.

The lack of experimental data on flow over rough walls and near to a separation point clearly hampers any serious attempt at establishing a definitive expression for $C$. An assessment on the applicability of $C$ on conditions to which it is not supposed to hold is thus a matter of utmost importance.

\section{Experiments}

Equations 1 and 4 will be tested against the smooth wall DNS data of $\mathrm{Na}$ and Moin (1998), NM, the smooth wall LDA data of Loureiro et al. (2007a, b), SS, and the two rough wall LDA datasets of Loureiro et al. (2008), RSA, RSB.

For a detailed description of the numerical simulations and of the water-channel experiments see the above references. Succinctly, the simulations of $\mathrm{Na}$ and Moin (1998) were conducted for flow over a flat, smooth surface with a prescribed adverse pressure gradient. The LDA measurements of Loureiro et al. (2007a, b, 2008) were conducted in a water-channel for flows over steep, smooth and rough hills. In all, 36 flow positions were characterized by Loureiro et al. (2007a, b, 2008): 13 for the SS condition, 10 for the RSA condition and 13 for the RSB condition. The main properties of the undisturbed profiles that define conditions NM, SS, RSA and RSB are shown in Table 1.

Table 1 Properties of the undisturbed profile

\begin{tabular}{lllll}
\hline Property & NM & SS & RSA & RSB \\
\hline Boundary layer thickness $(\delta, \mathrm{mm})$ & 42.8 & 100 & 90 & 100 \\
Displacement thickness $\left(\delta_{1}, \mathrm{~mm}\right)$ & 1.9 & 9 & 16 & 15 \\
External velocity $\left(U_{\delta}, \mathrm{m} \mathrm{s}^{-1}\right)$ & 4.19 & 0.0482 & 0.0497 & 0.3133 \\
Friction velocity $\left(u_{*}, \mathrm{~m} \mathrm{~s}^{-1}\right)$ & 0.2208 & 0.0028 & 0.0047 & 0.0204 \\
Displacement height $(d, \mathrm{~mm})$ & 0.0 & 0.0 & 2.1 & 2.0 \\
Roughness length $\left(z_{0}, \mathrm{~mm}\right)$ & 0.01 & 0.08 & 0.83 & 0.33 \\
Reynolds number $\left(R_{\delta}\right)$ & 11,446 & 4,772 & 4,425 & 31,023 \\
Reynolds number $\left(R_{z_{0}}\right)$ & 2.82 & 0.22 & 3.88 & 6.65 \\
\hline
\end{tabular}


The main characteristics of the laser Doppler system varied between experiments. Most measurements were carried out with a two-component Dantec system fitted with an Ar-ion tube laser that was set to operate in the backscatter mode. A Bragg cell unit was used to resolve flow direction. Typical uncertainties associated with the mean velocity data are $<0.002$ of $U_{\delta}$ (the free stream velocity). In regions of reverse flow, the uncertainties increase to about $0.003 U_{\delta}$. The hill was $60 \mathrm{~mm}$ high and followed a Witch of Agnesi profile. The roughness elements consisted of rigid rubber strips $3 \mathrm{~mm}$ wide by $3 \mathrm{~mm}$ high that were spaced by $9 \mathrm{~mm}$. The rough surface extended from $1.5 \mathrm{~m}$ upstream of the hill top to $1.5 \mathrm{~m}$ downstream.

\section{Results}

The applicability of Eq. 4 is assessed through the following procedure. First, parameters $u_{*}$, $\partial_{x} p$ and $C$ are estimated directly from Eq. 1 for given profiles of $u$ versus $z$. To denote all parameters estimated by this procedure, the subscript est is used. Next, parameters $u_{*}$ and $\partial_{x} p$ are again estimated but with $C$ given by Eq. 4 . These parameters are identified through the subscript par. The estimated (est) and parametrized (par) results are then compared with the directly calculated smooth surface values of $\mathrm{Na}$ and Moin (1998) and of Loureiro et al. (2007a, b). For flow over a rough surface, where directly measured values of $u_{*}$ are not available, results are only compared with each other.

To calculate the parameters for the rough surface flows (RSA and RSB), $d$ and $z_{0}$ have been kept constant and equal to the values determined in Loureiro et al. (2008) for the undisturbed velocity profiles. The very detailed analysis of Loureiro et al. (2008) argues that conditions RSA and RSB yield aerodynamically rough flows and explains how $d$ and $z_{0}$ are evaluated according to the gradient method of Clauser (1954) and Perry and Joubert (1963).

The same procedure described in Loureiro et al. (2008) was adopted here to estimate the parameters in Eqs. 1 and 4. Global optimization algorithms based on direct search methods were used. Despite their tendency to converge more slowly, direct search methods can be more tolerant to the presence of noise in the function and to constraints. Four different methods were used for the solution search: Nelder Mead, Differential Evolution, Simulated Annealing and Random Search. Only when all four methods furnished consistent results, with accuracy down to the sixth decimal place, was the search stopped.

The fitting results are illustrated by the selection of curves presented in Figs. 1-4. All shown curves were drawn with the estimated values of $C, C_{\text {est }}$. Curves were chosen so as to represent conditions upstream of the separation point and in the region of reversed flow for every flow condition.

For flow over a smooth wall, Figs. 1a and 2a, the viscous, fully turbulent and outer regions are well discerned in the upstream profiles. Equation 1 is observed to fit very well in the fully turbulent region, thus capturing the logarithmic-dominated shape of the local velocity profiles. In the regions of reverse flow, a large flow portion can be identified where Eq. 1 is observed to represent well the negative velocity profile. The data of Loureiro et al. (2007a, b) also show that a curve fit resulting from Eq. 1 represents very well the reverse flow velocity profile except nearest the wall where the viscous effects dominate.

The curve fits for flows over a rough surface are shown in Figs. 3 and 4, where the destruction of the viscous layer by the roughness elements is evident. The consequence is that the set of points to be fitted extends right down to the wall. Agreement between Eq. 1 and the data is very good, independent of the flow station, and whether the location is in regions of attached or reverse flow. 

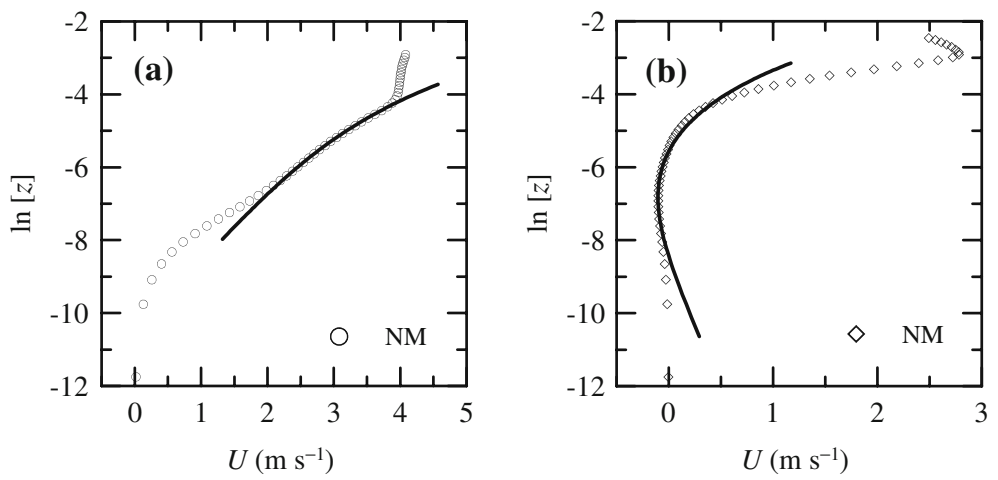

Fig. 1 Typical curve fits to the data of $\mathrm{Na}$ and Moin (1998). a Upstream profile at station $x / \delta_{i n}^{*}=100$. b Reverse flow region profile at station $x / \delta_{i n}^{*}=250 . z$ is plotted in $\mathrm{m}$
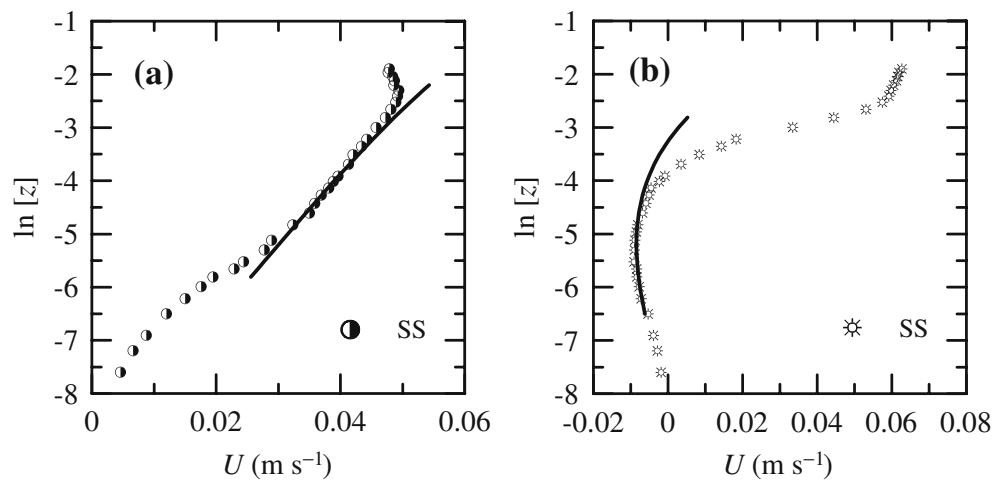

Fig. 2 Typical curve fits to the data of Loureiro et al. (2007a, b), SS condition. a Upstream profile at station $x / H=-12.5$. b Reverse flow region profile at station $x / H=3.75 . z$ is plotted in $\mathrm{m}$
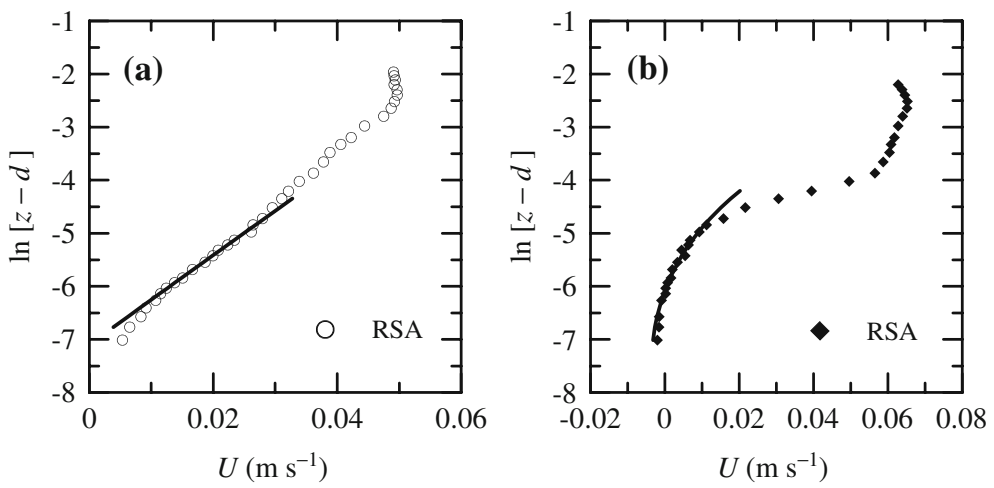

Fig. 3 Typical curve fits to the data of Loureiro et al. (2008), RSA condition. a Upstream profile at station $x / H=-5.8$. b Reverse flow region profile at station $x / H=1.31$. $z$ is plotted in $\mathrm{m} . z=$ vertical distance from the bottom of the roughness elements, $d=$ zero-plane displacement 

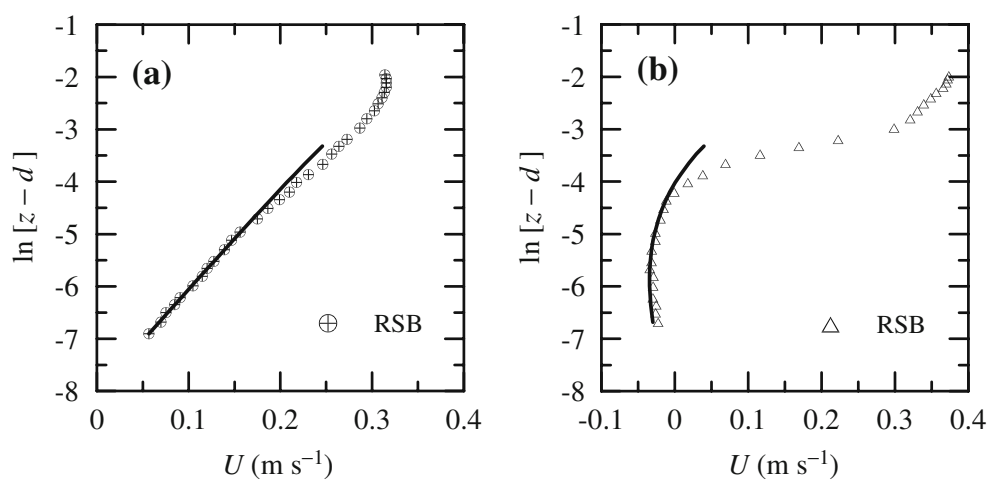

Fig. 4 Typical curve fits to the data of Loureiro et al. (2008), RSB condition. a Upstream profile at station $x / H=-17.87$. b Reverse flow region profile at station $x / H=3.06$. $z$ is plotted in $\mathrm{m} . z=$ vertical distance from the bottom of the roughness elements, $d=$ displacement height
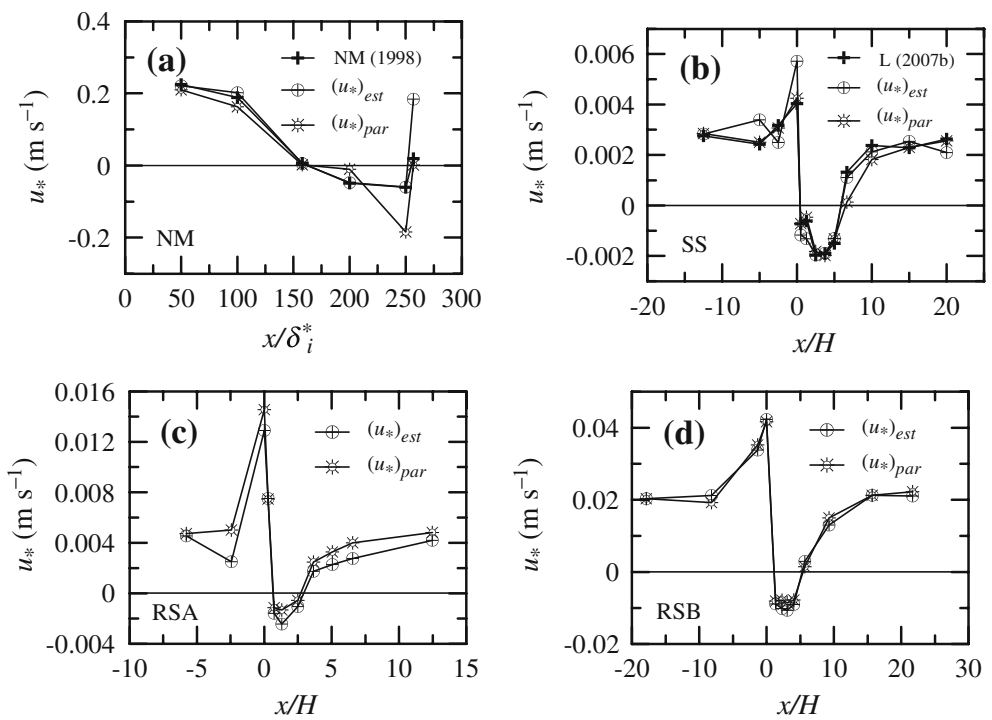

Fig. 5 Predictions of $u_{*}\left(\mathrm{~m} \mathrm{~s}^{-1}\right)$. ( $\left.u_{*}\right)_{e s t}$ stands for values of $u_{*}$ estimated directly from Eq. 1 with no parametrization of $C .\left(u_{*}\right)_{\text {par }}$ stands for values of $u_{*}$ estimated from Eq. 1 with $C$ parametrized through Eq. 4. a Data of Na and Moin (1998). b Data of Loureiro et al. (2007a, b), SS condition. c Data of Loureiro et al. (2008), RSA condition. d Data of Loureiro et al. (2008), RSB condition

Predictions of $u_{*}$ based on Eqs. 1 and 4 are shown in Fig. 5. For the smooth wall data, three sets of points are shown: predictions with estimated $C\left(=C_{e s t}\right)$ from Eq. 1, predictions with parametrized $C\left(=C_{\text {par }}\right)$ from both Eqs. 1 and 4, and the reference data of $\mathrm{Na}$ and Moin (1998) and of Loureiro et al. (2007a, b).

For Na and Moin (1998) (Fig. 5a), both predictions agree well with the DNS data, except at two positions close to the reattachment point. Actually, predictions with $C_{\text {est }}$ are almost exact for all the flow domain, except near the reattachment point where a very high value is found. The parametrization of $C$ yields slightly lower values of $u_{*}$ upstream of the 

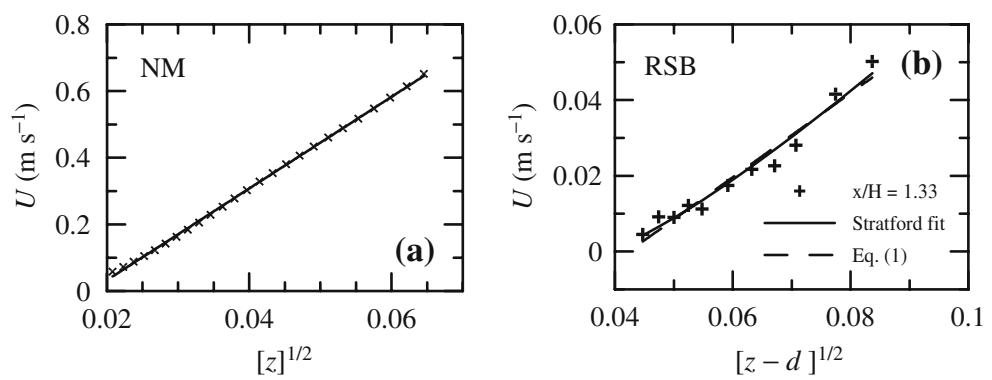

Fig. 6 Stratford profiles. $z$ is plotted in $\mathrm{m} ; U$ in $\mathrm{ms}^{-1}$. a Data of Na and Moin (1998). b Data of Loureiro et al. (2008), RSB condition

separation point, an almost exact prediction at the separation and reattachment points, and an uncharacteristic very low value just before the reattachment.

Predictions for the smooth wall data of Loureiro et al. (2007a, b) are shown in Fig. 5b. Upstream of the hill, at $x / H=-12.5$, both predictions coincide with the reference experimental value of $u_{*}$. On the upwind side of the hill and on the hill top, predictions with $C_{\text {par }}$ give an almost exact result. The use of $C_{\text {est }}$ overshoots $u_{*}$ at the base of the hill $(x / H=-5)$ and at the top $(x / H=0)$, and gives a lower value at $x / H=-2.5$. In the region of reverse flow, predictions through both procedures agree very well with the experimental data. Downstream of the hill, predictions with $C_{\text {par }}$ exhibit a slow recovery to the undisturbed values of $u_{*}$. Position $x / H=6.67$, in particular, shows the worst agreement. Use of $C_{\text {est }}$ results in very good predictions for $x / H \geq 6.67$.

The rough wall data of Loureiro et al. (2008) are shown in Fig. 5c and d. For the lower Reynolds number dataset (RSA condition), Eq. 4 gives consistently higher values of $u_{*}$ when compared with values obtained through a direct estimation of $C$ (Fig. 5c). The worst agreement occurs at position $x / H=-2.45$, the mid-length of the upstream slope of the hill. Results for the higher Reynolds number dataset (RSB condition) offer a very distinct perspective (Fig. 5d) on the usefulness of Eq. 4 . The differences between values of $u_{*}$ given by both procedures are almost absent. The parametrization works well even in regions where it is supposed to fail, that is, close to separation and attachment points.

Velocity profiles using coordinates as in Stratford are shown in Fig. 6 for conditions NM and RSB. The resulting best fits are given respectively by $U=13.7455 z^{1 / 2}-0.2420$ and $U=1.1120 z^{1 / 2}-0.0471$. Hence, in the limit $\tau_{w} \rightarrow 0$, the additive parameter $C$ is different from zero, in opposition to Eq. 4.

To better understand the role of $C$ on $u_{*}$ predictions, Figs. 5 and 7 must be considered. In general, higher values of $C$ result in higher values of $u_{*}$ and vice versa. Exceptions, however, can be noted for conditions NM (two points near to reattachment) and SS (top of the hill). For the rough wall conditions, RSA and RSB, no exception is recorded. Regarding the NM condition, both $C_{e s t}$ and $C_{\text {par }}$ follow the same trend and are close together (Fig. 7a). Upstream of the separation point, $C_{e s t}>C_{\text {par }}$ is satisfied, and in the region of reverse flow this inequality is inverted. The crosses denote values of $C$ calculated directly from Eq. 1 with values of $u_{*}$ and $\partial_{x} p$ given by the DNS data.

For flow over the smooth and rough hills, SS, RSA and RSB conditions, we have $C_{\text {par }}>$ $C_{\text {est }}$. Only two exceptions upstream of the hill top are observed (Fig. 7b, d). Clearly, the higher the values of $u_{*}$, the less sensitive the results are to variations in $C$. For the SS condition, large variations of $C$ on the upstream side of the hill and at the hill top are visible 

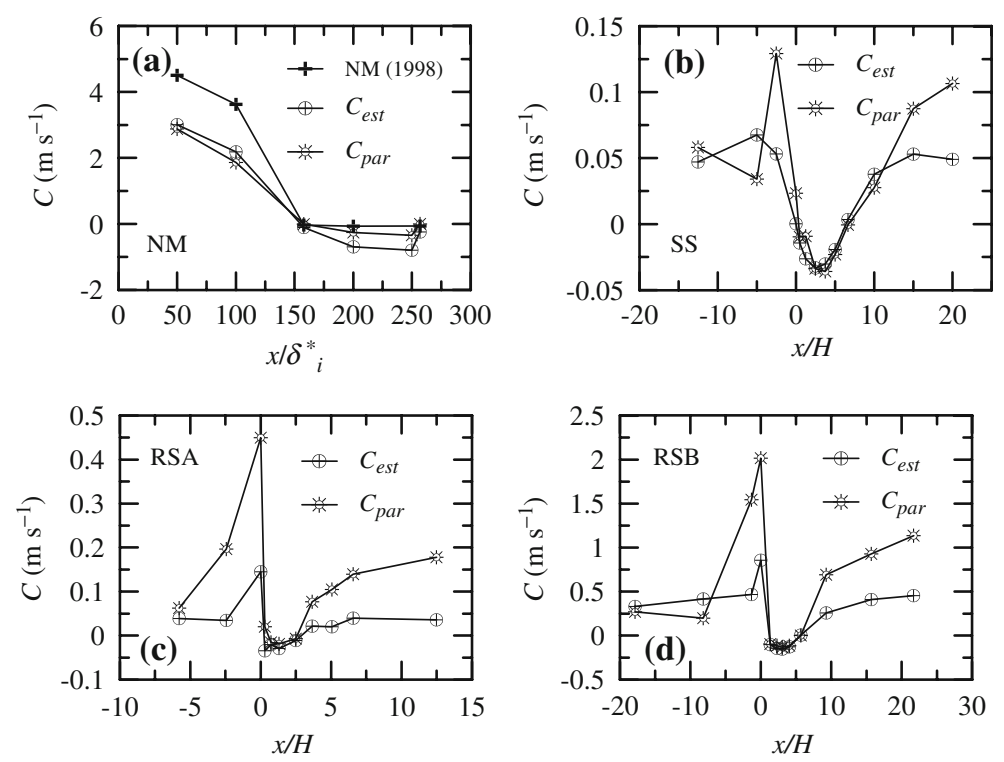

Fig. 7 Behaviour of the parametrization function $C\left(\mathrm{~m} \mathrm{~s}^{-1}\right)$. $C_{\text {est }}$ stands for values of $C$ estimated directly from Eq. 1. $C_{\text {par }}$ stands for values of $C$ estimated from Eq. 4. a Data of Na and Moin (1998). b Data of Loureiro et al. (2007a, b), SS condition. c Data of Loureiro et al. (2008), RSA condition. d Data of Loureiro et al. (2008), RSB condition

in the $u_{*}$ predictions. For conditions RSA and RSB, large changes in $C$ do not propagate significantly to $u_{*}$.

\section{Final Remarks}

The present work has investigated in detail the parametrization method proposed by Loureiro et al. (2008) that accounts for separation and roughness effects on the lower boundary condition for turbulent boundary layers. The overall agreement between the predictions of $u_{*}$ furnished by Eqs. 1 and 4 and the 'exact' data of $\mathrm{Na}$ and Moin (1998) and of Loureiro et al. (2007a, b, 2008) is very good, in particular, for condition SS.

In general, values furnished by $C_{\text {par }}$ are found to be larger than the estimated values, $C_{e s t}$, though the differences, however, are not transmitted in proportion to predictions of $u_{*}$. This is noticed for all conditions, particularly for RSA and RSB conditions. In fact, predictions of $u_{*}$ for condition RSB using $C_{\text {par }}$ and $C_{\text {est }}$ coincide almost exactly. An immediate implication is that $C_{p a r}$ can be used to describe the lower boundary condition in all flow regions, including regions away or near to a separation point.

The use of wall functions to specify the lower boundary condition has been greatly criticized in the past in regard to its apparent lack of validity outside the conditions of equilibrium flow, particularly close to flow separation. As an alternative approach, low Reynolds number turbulence models have been developed. The success of the latter approach relies necessarily on the deployment of very fine grids, capable of resolving the near-wall region to distances of a fraction of $v / u_{*}$. This requirement alone establishes such a large demand on computer cost as to make any attempt at the numerical simulation of environmental or industrial flows impractical. 
For flows over rough surfaces, the above discussion is out of place: the wall function approach is the only viable option. It is then just natural that many authors have sought extensions of the standard law-of-the-wall to rough walls. This is a very complex task in its own right. Very few authors, however, have dealt simultaneously with separation and roughness, and the present contribution helps to fill this gap. Together with Loureiro et al. (2008), this work forms a consistent theory on flow subject to separation and roughness effects, which might in the future be implemented in predictive numerical codes.

The present work has also provided valuable data on the mean properties of turbulent separated flows over rough hills, in particular, the wall shear stress, that can be used to validate numerical simulations of this flow problem.

Acknowledgements JBRL benefited from a Research Fellowship from the Brazilian Ministry of Science and Technology through Programme Prometro (Grant No 554391/2006-6). APSF is grateful to the Brazilian National Research Council (CNPq) for the award of a Research Fellowship (Grant No 306977/2006-0). The work was financially supported by CNPq through Grants No 477392/2006-7 and No 476091/2007-1 and by the Rio de Janeiro Research Foundation (FAPERJ) through Grants E-26/171.346/2005 and E-26/171.198/2003.

\section{References}

Arya SPS, Capuano ME, Fagen LC (1987) Some fluid modelling studies of flow and dispersion over twodimensional low hills. Atmos Environ 21:753-764

Athanassiadou M, Castro IP (2001) Neutral flow over a series of rough hills: a laboratory experiment. Boundary-Layer Meteorol 101:1-30

Britter RE, Hunt JCR, Richards KJ (1981) Air flow over a two-dimensional hill: studies of velocity speedup, roughness effects and turbulence. Q J Roy Meteorol Soc 107:91-110

Cheng H, Castro IP (2002) Near-wall flow development after a step change in surface roughness. BoundaryLayer Meteorol 105:411-432

Clauser FH (1954) Turbulent boundary layers in adverse pressure gradients. J Aeronaut Sci 21:91-108

Cruz DOA, Silva Freire AP (1998) On single limits and the asymptotic behaviour of separating turbulent boundary layers. Int J Heat Mass Transf 41:2097-2111

Cruz DOA, Silva Freire AP (2002) Note on a thermal law of the wall for separating and recirculating flows. Int J Heat Mass Transf 45:1459-1465

Garratt JR (1992) The atmospheric boundary layer. Cambridge University Press, Cambridge, 316 pp

Kaimal JC, Finnigan JJ (1994) Atmospheric boundary layer flows-their structure and measurement. Oxford University Press, New York, 289 pp

Loureiro JBR, Soares DV, Fontoura Rodrigues JLA, Pinho FT, Silva Freire AP (2007a) Water tank and numerical model studies of flow over steep smooth two-dimensional hills. Boundary-Layer Meteorol 122:343-365

Loureiro JBR, Pinho FT, Silva Freire AP (2007b) Near wall characterization of the flow over a twodimensional steep smooth hill. Exp Fluids 42:441-457

Loureiro JBR, Monteiro AS, Pinho FT, Silva Freire AP (2008) Water tank studies of separating flow over rough hills. Boundary-Layer Meteorol 129:289-308

Na Y, Moin P (1998) Direct numerical simulation of a separated turbulent boundary layer. J Fluid Mech 374:379-405

Ohba R, Hara T, Nakamura S, Ohya Y, Uchida T (2002) Gas diffusion over an isolated hill under neutral, stable and unstable conditions. Atmos Environ 36:5697-5707

Oke TR (1987) Boundary layer climates. Routledge, London, 435 pp

Perry AE, Joubert PN (1963) Rough-wall boundary layers in adverse pressure gradients. J Fluid Mech 17:193211

Simpson RL (1996) Aspects of turbulent boundary-layer separation. Prog Aero Sci 32:457-521

Snyder WH, Britter RE (1987) A wind-tunnel study of the flow structure and dispersion from sources upwind of three-dimensional hills. Atmos Environ 21:735-751

Stratford BS (1959) The prediction of separation of the turbulent boundary layer. J Fluid Mech 5:1-16

Wood N (1995) The onset of separation in neutral, turbulent flow over hills. Boundary-Layer Meteorol 76:137164 